\title{
The Clinical Value of the Self-Administered Kamath and Stothard's Questionnaire in the Diagnostics of Carpal Tunnel Syndrome
}

\author{
Ferenc Toth ${ }^{1}$, Elizabeth Kiss ${ }^{1}$, Csaba Marafko ${ }^{2}$, Jozsef Nemes ${ }^{2}$, Zsolt Hegedus ${ }^{2}$ \\ ${ }^{1}$ Barlborough Hospital, Practice Plus Group, Chesterfield, UK \\ ${ }^{2}$ Emersons Green Hospital, Practice Plus Group, Bristol, UK \\ Email: ^Ferenc.Toth@practiceplusgroup.com
}

How to cite this paper: Toth, F., Kiss, E., Marafko, C., Nemes, J. and Hegedus, Z. (2021) The Clinical Value of the Self-Administered Kamath and Stothard's Questionnaire in the Diagnostics of Carpal Tunnel Syndrome. Open Journal of Therapy and Rehabilitation, 9, 70-82.

https://doi.org/10.4236/ojtr.2021.92006

Received: March 2, 2021

Accepted: May 23, 2021

Published: May 26, 2021

Copyright $\odot 2021$ by author(s) and Scientific Research Publishing Inc. This work is licensed under the Creative Commons Attribution International License (CC BY 4.0).

http://creativecommons.org/licenses/by/4.0/

\section{(c) (i) Open Access}

\begin{abstract}
The clinician-administered Kamath and Stothard's questionnaire is a recommended tool for assessing Carpal Tunnel Syndrome (CTS). The authors investigated whether the self-administered Kamath and Stothard's questionnaire (KSQ) can categorize the severity of CTS according to the Nerve Conduction Study (NCS) and predict the outcome of Carpal Tunnel Release (CTR). One hundred and forty-two consecutive patients were enrolled in this prospective study, who completed the KSQ and were referred for NCS. Symptom relief after CTR was considered as the reference standard. KSQ's scores were compared with the severity of CTS confirmed by NCS and the effectiveness of the surgery, Sensitivity and Positive Predictive Value (PPV) of the KSQ was calculated. The sensitivity of KSQ in each NCS severity category (mild, moderate, and severe CTS) was low (11.9\% - 50.0\%), however, the sensitivity and PPV of KSQ in the moderate + severe CTS category was $88.9 \%$ and $95.5 \%$. The KSQ score of 5 or greater was found to be correlated with a successful CTR (sensitivity $92.8 \%$, PPV of $90.2 \%$ ). The study revealed that the KSQ with a score of 5 or greater is able to distinguish the mild conditions from moderate and severe ones. The KSQ's score of 5 or greater also can predict the successful outcome of CTR. The authors believe that in addition to reducing the duration and cost of diagnostics, the KSQ can also reduce patient appointments and clinic time. Application of the KSQ may be of paramount importance in the current Covid pandemic era.
\end{abstract}

\section{Keywords}

Carpal Tunnel Syndrome, Nerve Conduction Study, Self-Administered Questionnaire 


\section{Introduction}

The British Society for Surgery of the Hand (BSSH) published their recommendation for the treatment of Carpal Tunnel Syndrome (CTS) in 2011 [1]. The BSSH protocol divided CTS into 3 severity levels (mild, moderate, and severe CTS) based on its signs and symptoms and defined treatment according to the severity. In the case of mild and moderate CTS, conservative management is the first advocated treatment (e.g., night wrist splint and/or steroid injection). Carpal Tunnel Release (CTR) is advised for those patients who are suffering from severe CTS or underwent 6 - 12 weeks of unsuccessful conservative treatment [1].

The revised guideline for the diagnosis and treatment of CTS was published in “The Royal College of Surgeons (RCS) Commissioning Guide" in 2017 [2]. This guidance suggests that mild CTS should be treated with a trial of evidence-based conservative management while those with moderate or severe CTS could be considered for surgery [2].

According to these guidelines, the treatment of CTS is based on its severity.

In order to determine the severity of CTS, a detailed clinical history and comprehensive physical examination is indispensable [1] [2]. However, the patient's history and clinical findings occasionally are unable to determine the severity of CTS and/or distinguish the CTS from other neurological disorders (e.g.: irritation of cervical nerve root, neuropathy, etc.) [3] [4].

To increase the accuracy of diagnostics, the Nerve Conduction Study (NCS) is the most frequently applied diagnostic method in everyday practice [5]. However, the diagnostic value of the NCS is controversial [6] [7]. Few authors consider the NCS as the "gold standard" for a true diagnosis of CTS [3] [4]. However, several investigators called into question the utility of NCS as a diagnostic criterion standard [8] [9] [10]. Sonoo et al. (2018) concluded in their study that lacking a gold standard for diagnosis of CTS, all reasonable diagnostic measures including symptoms/signs and NCS should be used to increase diagnostic accuracy [11].

In recent years there has been an increase in the use of self-administered questionnaires (i.e., Boston Carpal Tunnel Questionnaire, The Disability of Arm, Shoulder and Hand Questionnaire, etc.) for the assessment of the severity of symptoms and functional status of patients with CTS. These papers concluded that self-administered questionnaires are reproducible, internally consistent, valid, and responsive to clinical change [5] [12] [13].

Kamath and Stothard (2003) recommended the use of the scored clinical questionnaire (Figure 1) to diagnose the CTS [4] [13]. This questionnaire was completed by an experienced hand surgeon in their study. It was concluded that using the score of 5 as the threshold for CTS, the sensitivity (85\%) and positive predictive value (90\%) of the questionnaire is nearly identical to these values of NCS (sensitivity 92\%, positive predictive value 92\%), therefore, the Kamath and Stothard's questionnaire can replace NCS in the initial assessment of CTS [4]. 
1. Has pain in the wrist woken you at night?

$$
\text { Yes } 1 \quad \text { No } 0
$$

2. Has tingling and numbness in your hand woken you during the night?

$$
\text { Yes } 1 \quad \text { No } 0
$$

3. Has tingling and numbness in your hand been more pronounced first thing in the morning?

Yes $1 \quad$ No 0

4. Do you have any trick movements to make the tingling, numbness go from your hands? Yes $1 \quad$ No 0

5. Do you have tingling and numbness in your little finger any time? Yes $0 \quad$ No 3

6. Has tingling and numbness presented when you were reading a news paper, steering a car or knitting? Yes $1 \quad$ No 0

7. Do you have any neck pain?

$$
\text { Yes - } 1 \quad \text { No } 0
$$

8. If applicable has the tingling and numbness in your hand been severe during pregnancy?

$$
\text { Yes } 1 \quad \text { No }-1 \quad \text { N/A } 0
$$

9. Has it helped the tingling and numbness on wearing a splint on your wrist?

$$
\text { Yes } 2 \text { No } 0 \quad \text { N/A } 0
$$

Figure 1. Kamath and Stothard's scored questionnaire.

The authors suggested the utilization of this questionnaire since it gives major time, personnel, and cost benefits [4].

The effectiveness of the clinician-completed Kamath and Stothard's questionnaire was analyzed in additional studies [5] [12] [14]. However, the clinical value of Kamath and Stothard's questionnaire as a "self-administered questionnaire" (KSQ) has not been comprehensively investigated.

It was also not examined whether the KSQ can categorize the CTS similar to the NCS according to its severity (mild, moderate, and severe CTS) or if the KSQ score can predict the effectiveness of surgery (CTR).

The primary goal of our multicenter-prospective study is to determine whether the KSQ can predict the severity of CTS compared to the result of NCS. Our secondary goal is to establish if the KSQ score can predict the outcome of CTR surgery.

\section{Methods and Materials}

Patients referred by primary care physicians (GPs) between January 2016 and January 2017 with a diagnosis of suspected CTS were enrolled in the study. Patients were seen at the hand clinic of two secondary care hospitals. The inclusion criteria of the study were as follows: at least 2 clinical signs and/or symptoms of CTS, including the pain-altered sensation of the thumb/index-/middle- and ring finger, positive Phalen test, positive Tinnel test, thenar atrophy. Electrodiagnos- 
tic evidence of CTS in itself meant inclusion into the study. Exclusion criteria contained: the inability of data gathering, coexisting cervical radiculopathy, brachial plexopathy, proximal median nerve mononeuropathy, clinical and electrophysiological signs of polyneuropathy, rheumatoid arthritis, diabetes mellitus, underactive thyroid, multiple sclerosis, renal and heart failure. Verbal informed consent was obtained from all patients enrolled in the study

A total of four surgeons (Consultant Orthopedic Surgeons with a special interest in Hand Surgery and working in secondary care) participated in the study, who followed the principle of "continuity of care" when treating patients. That is, pre-assessment, surgery and post-operative follow-up were performed by the same surgeon. Patients who underwent conservative treatment were followed up by an experienced physiotherapist. The physiotherapist assessed the outcome of the conservative treatment, such as taking the patient's clinical history, repeating all the physical examinations that were performed during the pre-assessment and completing the Post-treatment questionnaire.

Diagnostics, treatment and follow-up of the patients were performed according to the defined protocol below.

\subsection{Diagnostic Protocol}

All patients underwent a clinical history and physical examination at the initial assessment and were subsequently referred for NCS. Patients also completed the KSQ during the first appointment. The bilateral hand symptoms were evaluated individually, the NCS and KSQ were completed to each hand separately. The clinical history and physical examination were repeated at every follow-up.

The clinical history was performed according to the recommendation of BSSH (Figure 2). At the physical examination, in addition to the general examination of the hand, the following CTS-specific tests were performed: Tinel sign, Phalen test, Abductor Pollicis Brevis muscle power test, and observation of thenar muscle's wastage.

On the basis of clinical history and physical examination the CTS was classified into three categories (mild, moderate, or severe CTS) and was treated accordingly. The characteristics of mild CTS were intermittent paresthesia, nocturnal waking and pain in the hand/wrist. Moderate CTS was described as constant paresthesia, interference with activities of daily living, reversible numbness/pain and weakness/clumsiness of the hand. CTS was considered severe in the presence of symptoms, such as diminished sensation, disabling pain, wasting of the thenar muscle, and weakness of the Abductor Pollicis Brevis/Opponens Pollicis muscle.

The NCS was undertaken by two separate neuro-physiologists (one neurophysiologist at each hospital using the same NCS system) who were blinded to the KSQ score. The NCS was performed under standardized conditions in order to eliminate technical and physiological errors. The electrophysiological severity of CTS was established according to the Stevens Classification System (Figure 3) [15]. 


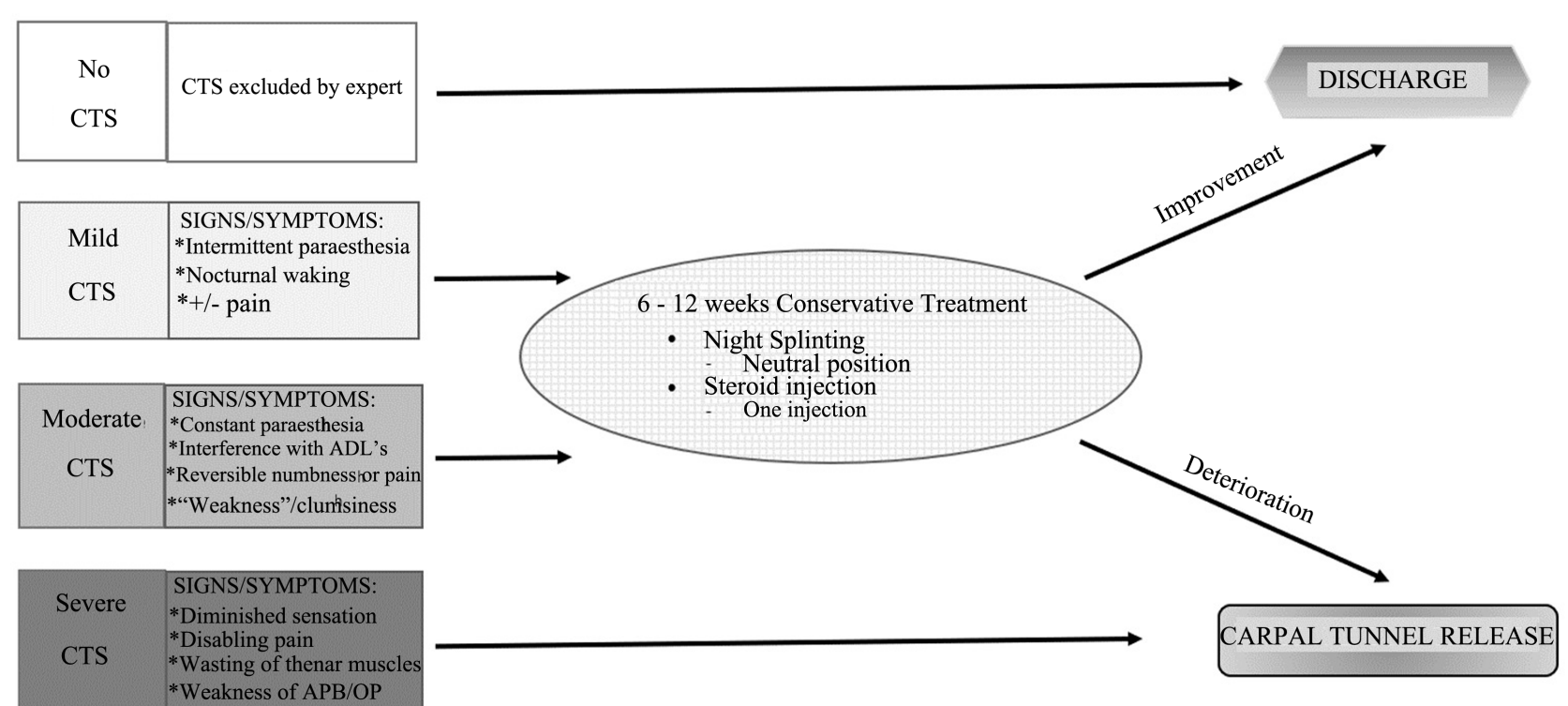

Figure 2. The carpal tunnel syndrome's signs/symptoms and treatment based on the protocol of British Society for Surgery of the Hand. CTS: Carpal Tunnel Syndrome, ADL: Activity of Daily Living, APB: Abductor Pollicis Brevis, OP: Opponens Pollicis.

\begin{tabular}{|c|c|c|}
\hline \multirow{2}{*}{$\begin{array}{c}\text { Electrophysiological } \\
\text { severity of CTS }\end{array}$} & \multicolumn{2}{|c|}{ Stevens Classification } \\
\hline & Sensory NCS & Motor NCS \\
\hline $\begin{array}{l}\text { MILD } \\
\text {-At least three of the } \\
\text { following Sensory and } \\
\text { Motor Nerve } \\
\text { Conduction: }\end{array}$ & $\begin{array}{l}\text { 1. } 14 \mathrm{~cm} \text { wrist stimulation, peak } \\
\text { latency }>3.7 \mathrm{~ms} \\
\text { 2. } 14 \mathrm{~cm} \text { wrist stimulation, peak latency: } \\
\text { proximal } 7 \mathrm{~cm}>\text { distal } 7 \mathrm{~cm} \\
\text { 3. Transcarpal } 5 \mathrm{~cm} \text { short-segment latency: } \\
\text { onset latency }>1.3 \text { ms, peak latency }>1.5 \mathrm{~ms} \\
4.14 \mathrm{~cm} \text { SNAP amplitude: } 16-20 \mu \mathrm{V} \\
\text { 5. Conduction block greater than } 50 \% \text { in wrist } \\
\text { palm stimulation If } 14 \mathrm{~cm} \text { stimulation } \\
\text { amplitude } \geq 20 \mu \mathrm{V}\end{array}$ & $\begin{array}{l}\text { 6. Distal latency }>4.2 \mathrm{~ms} \\
\text { 7. CAMP amplitude: } \\
4.1-4.5 \mathrm{mV}\end{array}$ \\
\hline $\begin{array}{l}\text { MODERATE } \\
\text {-Mild PLUS at least } \\
\text { two of the following: }\end{array}$ & $\begin{array}{l}\text { 1. Wrist stimulation }(14 \mathrm{~cm}) \text { SNAP } \\
\text { amplitude } \geq 6-15 \mu \mathrm{V} \\
\text { 2. Conduction block greater than } 50 \% \text { in wrist } \\
\text { and palm stimulation If SNAP } \geq 10 \mu \mathrm{V} \text { with } \\
14 \mathrm{~cm} \text { wrist stimulation }\end{array}$ & $\begin{array}{l}\text { 3. CAMP amplitude: } \\
2.1-4 \mathrm{mV}\end{array}$ \\
\hline SEVERE & & \\
\hline $\begin{array}{l}\text {-Moderate PLUS } \\
\text { one of the following: }\end{array}$ & 1. SNAP amplitude $\leq 5 \mu \mathrm{V}$ & 2. CAMP amplitude $\leq 2 \mathrm{mV}$ \\
\hline
\end{tabular}

Figure 3. Stevens Classification: Electrodiagnostic criteria to determine the severity of CTS. CTS: Carpal Tunnel Syndrome.

\subsection{Treatment Protocol}

Patients were treated according to the BSSH protocol (Figure 2).

Patients with a diagnosis of "No CTS" were referred back to the GP's care without treatment.

Of those patients who suffered from mild or moderate CTS, conservative treatment (night wrist splint and/or steroid injection) was introduced for 6 weeks. The clinical history and physical examination were repeated upon com- 
pletion of conservative treatment. If 6 weeks of conservative treatment failed to improve the patient's symptoms, i.e., the score of the post-treatment questionnaire was less than 6 (Figure 4), CTR was performed. Patients whose symptoms were significantly reduced or resolved with conservative treatment, i.e., the posttreatment score was 6 or greater were discharged to the care of the GP.

The patients who demonstrated severe CTS were booked for CTR, which was performed as a "day-case" under local anesthesia.

\subsection{Post-Treatment Questionnaire}

The post-treatment questionnaire (Figure 4) was used to determine the efficacy of the conservative/surgical treatment. It was compiled based on the signs and symptoms contained in the BSSH's protocol.

Since the post-treatment questionnaire was based on the BSSH's protocol and used by multiple experts (surgeons, physiotherapist) we considered it as valid without further validation.

The post-treatment questionnaire was completed by the surgeon (after CTR) or physiotherapist (after conservative treatment) at the 6 weeks appointment. When the patient did not attend, the post-treatment questionnaire was posted and the patient was asked to complete and return it. If no response was received, the patient was contacted by telephone (within 3 weeks after the missed appointment) to enable completion of the questionnaire.

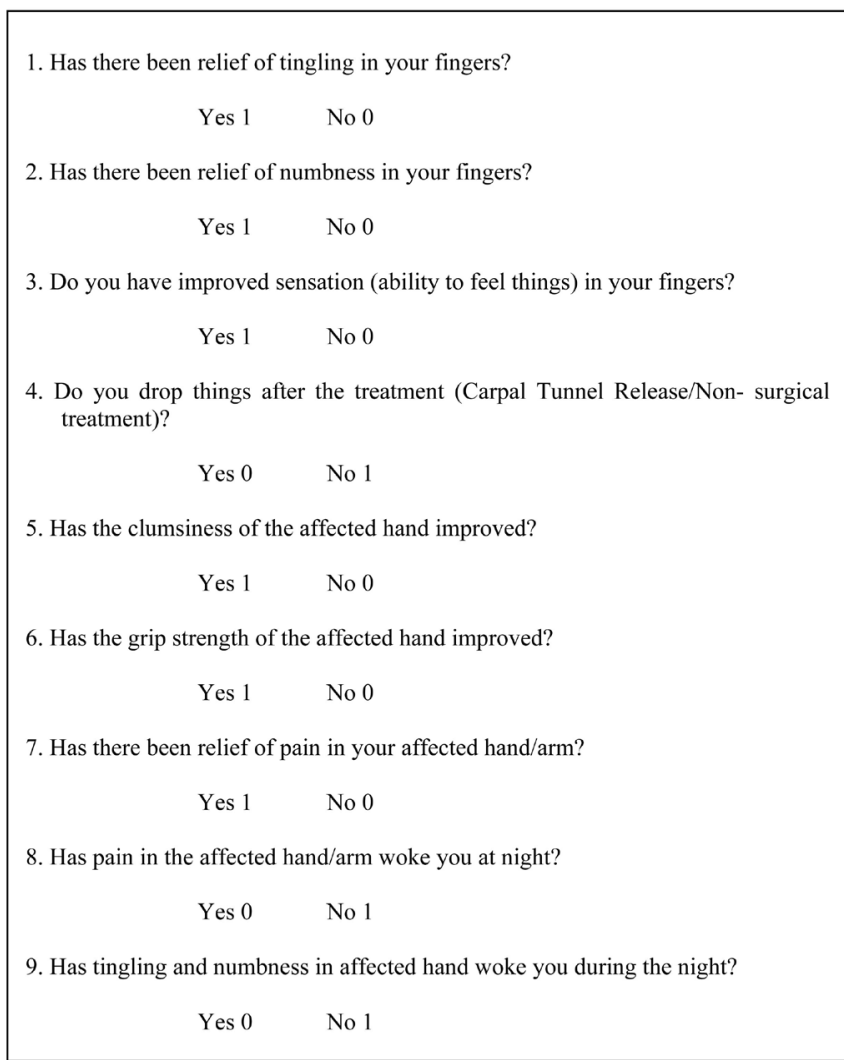

Figure 4. Post- treatment questionnaire. 


\subsection{Statistical Analysis}

The reference standard for a diagnosis of CTS was the significant relief of symptoms (i.e., post-treatment questionnaire score $\geq 6$ ) after surgical decompression of the median nerve (CTR). Therefore, patients who underwent conservative treatment were excluded from the statistical analysis.

The "cut off" value of the KSQ score for CTSs successfully treated with surgery was calculated using the mean-KSQ score, the standard deviation and $95 \%$ confidence interval. The Microsoft Excel program was used to calculate the "cut off" value:

NORM.INV (Probability, Mean, Standard deviation).

To assess the ability of the KSQ score to predict the electrophysiological severity of CTS, comparison of NCS classification and KSQ score was performed. The sensitivity and PPV of KSQ were calculated in the mild, moderate, and severe CTS groups. The patients were divided into 2 groups according to the above mentioned "cut off" value.

To establish the capability of KSQ to predict the surgical outcome, the success of surgery and KSQ score were compared. Patients were grouped again according to the "cut off" value. The outcome of surgical treatment was classified into "symptoms improved" and "symptoms not improved" groups according to the score of the post-treatment questionnaire. The post-treatment score of greater than or equal to 6 was considered to represent the successful surgical treatment.

Specificity and negative predictive value of KSQ could not be determined as a population without CTS was not included in the study.

\section{Results}

One hundred and forty-two patients with 159 suspected CTS were enrolled in the study (Figure 5). The average age of the patients was 59 years $(25-90)$ and the ratio of men to women was 53:89. Seventeen patients presented with bilateral

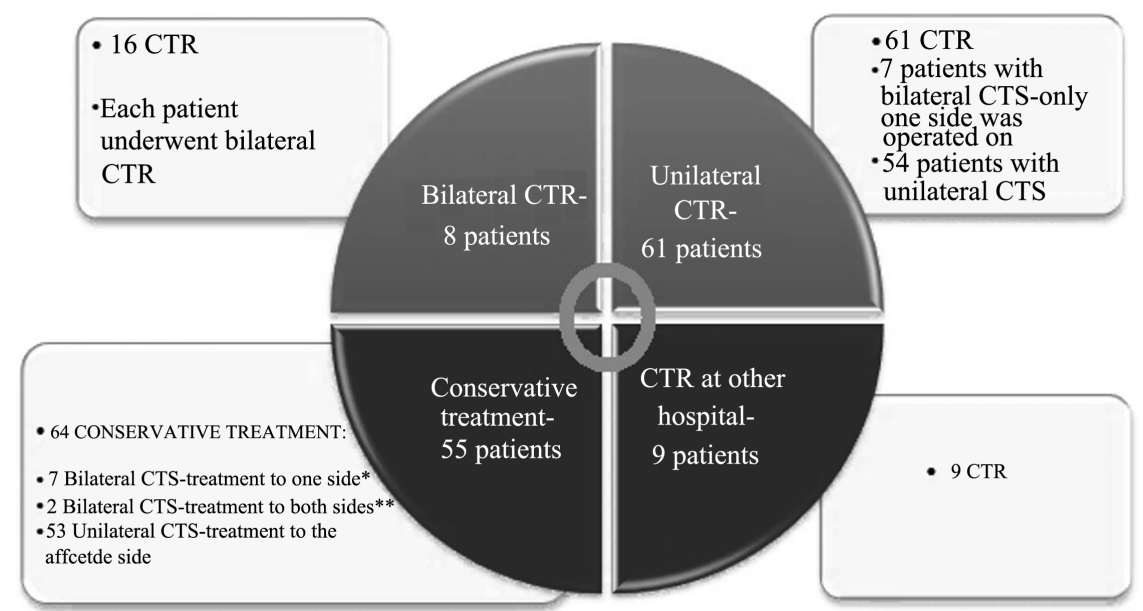

Figure 5. Distribution and treatment of patients with CTS according to BSSH protocol. CTS: Carpal Tunnel Syndrome, BSSH: British Society for Surgery of the Hand, CTR: Carpal Tunnel Release. 
CTS, 116 patients had unilateral CTS, the diagnosis of CTS was unconfirmed in 9 patients. Of the 17 patients with bilateral CTS, 8 underwent bilateral surgery, 7 unilateral CTR and 2 were treated conservatively.

Sixty-nine operated patients (77 CTR) were included in the statistical analysis. Nine patients (9 CTR), whose surgery was performed at another hospital, were excluded from statistical analysis due to the lack of postoperative data.

A comparison of KSQ scores with the incidence and electrophysiological severity of CTS among patients who underwent CTR showed that the overall incidence of CTS was significantly higher in patients with KSQ score of 6 (14 CTS), 7 (19 CTS), 8 (17 CTS) and 9 (13 CTS) (Figure 6).

An outstanding number of moderate and severe CTS cases were observed in patients with KSQ score 5, 6, 7, 8, and 9. The moderate and severe CTS occurred almost equally in patients with KSQ score of 5, 6, 7, 8, and 9. Mild CTS was observed in low numbers for almost all KSQ scores categories (Figure 6).

The calculation of the "cut off" value of the KSQ score for the CTSs that underwent successful surgery showed the following results; mean KSQ score: 7.17143, standard deviation: 1.61489 , "cut off" score: 4.51517 . As the latter is required to be a whole number it was rounded up to 5 , which was considered as a "cut off" score to predict the success of surgery.

To assess the ability of the KSQ to predict the electrophysiological severity of CTS, comparison of KSQ scores for each CTS severity category was performed. The analysis revealed low sensitivity values $(11.9 \%-50.0 \%)$ and high PPV $(80.0 \%-100.0 \%)$ in each severity categories (Table 1). However, when the moderate- and severe CTS cases were combined, the sensitivity value proved to be $88.9 \%$ and a PPV showed the value of $95.5 \%$ (Table 2). The sensitivity value and PPV for mild CTS cases were found to be $11.9 \%$ and $80 \%$, respectively.

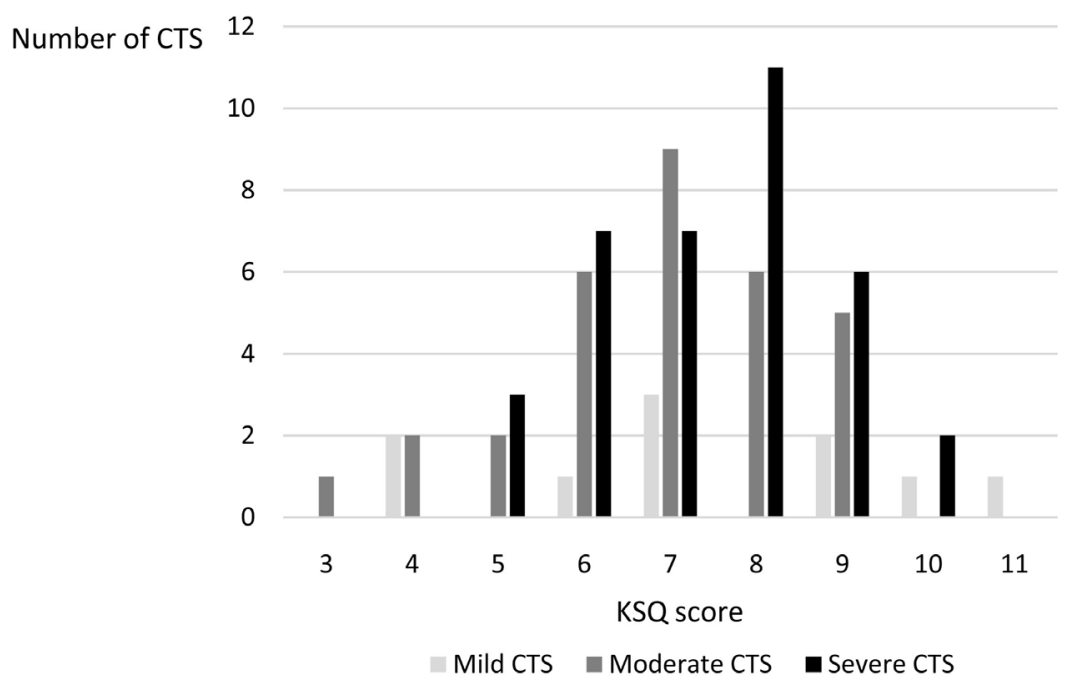

Figure 6. Distribution of KSQ scores with the incidence and severity of CTS confirmed by NCS among patients who underwent CTR $(n=77)$. CTS: Carpal Tunnel Syndrome, CTR: Carpal Tunnel Release, KSQ: Self-administered Kamath and Stothard's questionnaire, NCS: Nerve Conduction Study. 
Table 1. Comparison of KSQ scores and all NCS classifications of operated cases; Sensitivity and Positive Predictive Value of KSQ $(n=77)$. CTS: Carpal Tunnel Syndrome, KSQ: Self-administered Kamath and Stothard's questionnaire, NCS: Nerve Conduction Study.

\begin{tabular}{|c|c|c|c|c|c|}
\hline \multicolumn{6}{|c|}{ Sensitivity and Positive Predictive Value of KSQ according to CTS severity } \\
\hline \multicolumn{2}{|c|}{$\mathrm{KSQ} \geq 5 \mid<5$} & \multicolumn{2}{|c|}{$\mathrm{KSQ} \geq 5 \mid<5$} & \multicolumn{2}{|c|}{$\mathrm{KSQ} \geq 5 \mid<5$} \\
\hline \multicolumn{2}{|l|}{ Mild CTS } & \multicolumn{2}{|c|}{ Moderate CTS } & \multicolumn{2}{|c|}{ Severe CTS } \\
\hline Positive & $8 \mid 2$ & Positive & $28 \mid 3$ & Positive & $36 \mid 0$ \\
\hline Negative & $64 \mid 3$ & Negative & $44 \mid 2$ & Negative & $36 \mid 5$ \\
\hline Sensitivity: & $11.9 \%$ & Sensitivity: & $38.8 \%$ & Sensitivity: & $50.0 \%$ \\
\hline $\begin{array}{c}\text { Positive } \\
\text { Predictive Value: }\end{array}$ & $80.0 \%$ & $\begin{array}{c}\text { Positive } \\
\text { Predictive Value: }\end{array}$ & $90.3 \%$ & $\begin{array}{c}\text { Positive } \\
\text { Predictive Value: }\end{array}$ & $100.0 \%$ \\
\hline
\end{tabular}

Table 2. KSQ scores of mild-compared to moderate + severe CTS confirmed by NCS; sensitivity and positive predictive value of KSQ $(n=77)$. CTS: Carpal Tunnel Syndrome, KSQ: Self-administered Kamath and Stothard's Questionnaire, NCS: Nerve Conduction Study.

\begin{tabular}{cccc}
\hline \multicolumn{2}{c}{ Sensitivity and Positive Predictive Value of KSQ: Mild vs. Moderate + Severe CTS } \\
\hline \multicolumn{2}{c}{ KSQ } & \multicolumn{2}{c}{ KSQ } \\
$\geq 5 \mid<5$ & \multicolumn{3}{c}{ Moderate + Severe CTS } \\
Mild CTS & $8 \mid 2$ & Positive & $64 \mid 3$ \\
Positive & $64 \mid 3$ & Negative & $8 \mid 2$ \\
Negative & $11.9 \%$ & Sensitivity: & $88.9 \%$ \\
Sensitivity: & $80.0 \%$ & Positive Predictive Value: & $95.5 \%$ \\
Positive Predictive Value: & & & \\
\hline
\end{tabular}

Seventy CTRs were successful as these patients reported significant post-surgery improvement (score of post-treatment questionnaire $\geq 6$ ). Seven patients showed no improvement according to post-treatment scores (Figure 7). Their average post-treatment score was 3 .

Analysis of the accuracy of a KSQ score of 5 or greater to determine the success of CTR revealed a sensitivity value of $92.8 \%$ and PPV of $90.2 \%$ (Table 3 ).

\section{Discussion}

The application of questionnaires in the diagnostics of CTS has become more popular recently [4] [5] [7] [12].

One of the important requirements for questionnaires currently in use is that they are able to quantify the severity of CTS. Quantifying the severity of CTS simplifies the designation of treatment, facilitates objective monitoring of a treatment's effectiveness and provides an opportunity to perform objective comparative analyses [2]. The further important requirement for modern questionnaires is their applicability for web-based use [16].

Kamath and Stothard (2003) developed and tested a clinician-administered scored questionnaire for the diagnosis of CTS [4]. The authors found that 


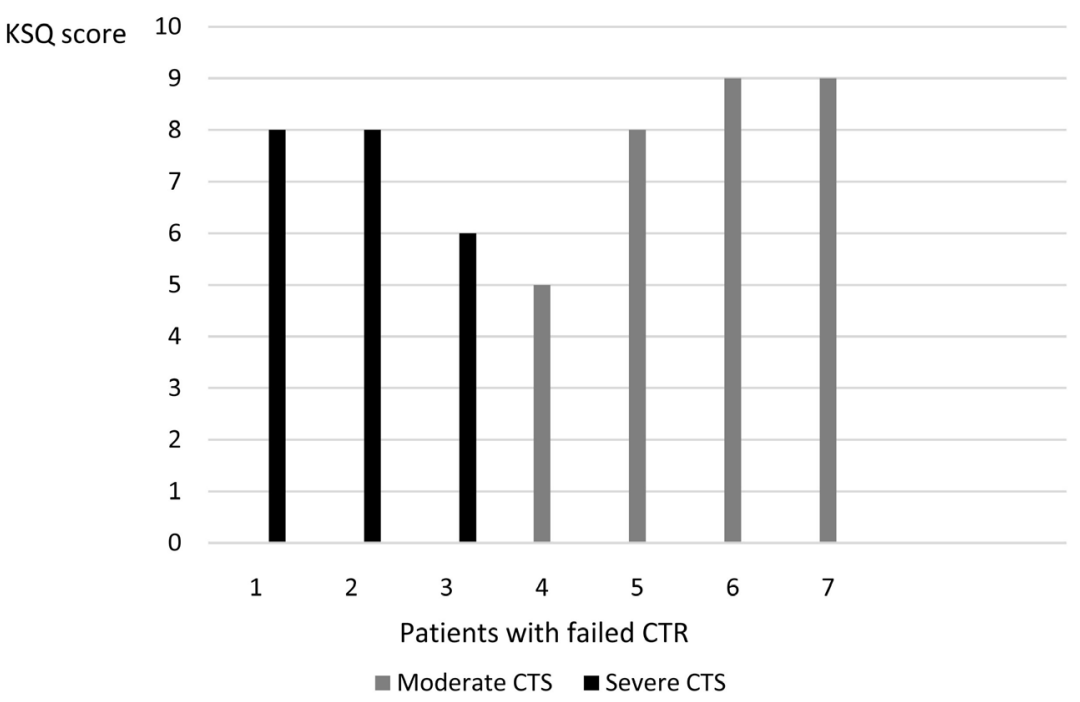

Figure 7. KSQ scores and severity of CTS (based on NCS findings) at failed CTR $(n=7)$. CTS: Carpal Tunnel Syndrome, CTR: Carpal Tunnel Release, KSQ: Self-administered Kamath and Stothard's questionnaire, NCS: Nerve Conduction Study.

Table 3. Post-treatment questionnaire results after CTR; Sensitivity and Positive Predictive Value of KSQ $(n=77)$. Symptom improvement measured by a score of $\geq 6$ of the post-treatment questionnaire. CTR: Carpal Tunnel Release, KSQ: Self-administered Kamath and Stothard's questionnaire.

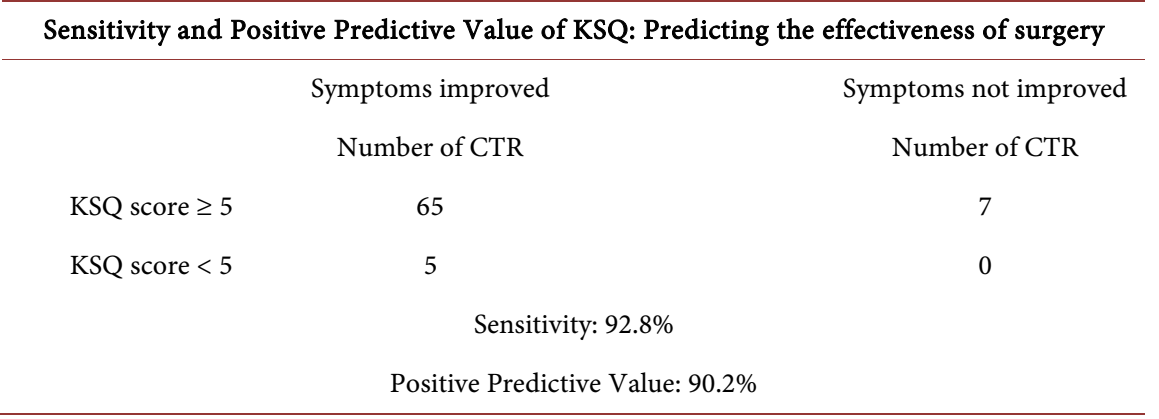

patients with scores of 5 or greater are suitable candidates for successful surgical decompression. The authors concluded that due to $85 \%$ sensitivity and $90 \%$ positive predictive value, the questionnaire can replace NCS in the diagnostics of CTS [4].

In another study, Edwards and Frampton (2014) compared the Kamath and Stothard's questionnaire scores with the NCS findings [12]. They reported that, of patients who had positive NCS, $82 \%$ had scored 5 or more. They suggested that patients scoring 7 or greater should not be offered confirmatory NCS due to $100 \%$ sensitivity and specificity of Kamath and Stothard's questionnaire [12].

Sangram et al. (2019) conducted a prospective study aimed to assess if the Kamath and Stothard's questionnaire could accurately predict positive NCS results when diagnosing CTS [5]. Their outcome was similar to the results of Kamath and Stothard [4]: considering 5 or greater scores as a positive questionnaire result, a sensitivity of $80 \%$, specificity of $70 \%$ and a positive predictive 
value of $90 \%$ were found for positive NCS [5].

It is generally accepted that self-administered/patient completed questionnaires have limitations, particularly with regard to misunderstanding of the questions subsequently resulting in the possibility of less accurate or meaningful answers [16]. Therefore, the novel aim of our study was to evaluate the clinical value of the self-administered Kamath and Stothard's questionnaire in the diagnostics of CTS.

In our study, the analysis of correlation between the electrophysiological severity of CTS and the KSQ score revealed that different categories of severity of CTS cannot be definitively distinguished by the KSQ. However, the KSQ score of 5 or greater can reliably distinguish mild CTS (sensitivity: $11.9 \%$, PPV: $80.0 \%$ ) from moderate + severe (sensitivity: $88.9 \%$, PPV: $95.5 \%$ ), which would require conservative or surgical treatment respectively.

The study also showed that the KSQ score of 5 or greater is a reliable indicator for the successful CTR surgery (sensitivity: 92.8\%, PPV: 90.2\%).

The authors propose that a KSQ score of 5 or greater suggests clinically and electrophsiologically proved moderate or severe CTS. Since the RCS advises surgery for moderate and severe CTS [2], these patients can undergo successful CTR without confirmatory NCS.

The authors believe that the application of KSQ can reduce the necessity of NCS confirming a diagnosis of CTS, thereby reducing the duration and cost of preoperative assessment. The data included in the KSQ assists the clinician to establish the clinical diagnosis, standardizes the record keeping, and facilitates the further processing of data. Using KSQ can reduce the number of appointments and shorten the time spent at the preassessment clinic. The importance of these benefits is particularly heightened in the current Covid pandemic era.

To our knowledge, our study is the first to investigate and show the accuracy of Kamath and Stothard's questionnaire as a self-administered tool determining the severity of CTS. While previous studies considered the outcome of the NCS as a "positive" or "negative" result, we evaluated the NCS's result according to its severity (mild, moderate and severe).

The authors recognize the limitations of their study with regard to patients for whom CTS has not been confirmed or have undergone conservative management. The data from these patients were not analyzed, therefore, the threshold of the KSQ score for the negative NCS and/or exclusion of CTS was not determined.

Further investigation of the psychometric properties of KSQ would help to establish whether this questionnaire could be used as web-based tool for the "fast-track" assessment of CTS [16]. Our findings may also provide a base for further studies, for example the KSQ could be incorporated into the further diagnostic protocol of CTS.

\section{Conclusion}

The novel finding of our prospective study is that the self-administered Kamath and Stothard's questionnaire (KSQ) with a score of 5 or greater is able to clearly 
distinguish the electrophysiologically confirmed mild CTS from moderate and severe ones (sensitivity: $88.9 \%$ PPV: $95.5 \%$ ). Another new finding of our study is that a KSQ score of 5 or greater can predict the successful outcome of CTR (sensitivity: 92.8\%, PPV: 90.2\%). Our findings suggest that the KSQ is a reliable tool for the assessment of patients with clinically suspected CTS and might be incorporated as an additional test in the diagnostic protocol recommended by The Royal College of Surgeons. Patients with a KSQ score of 5 or greater for whom there is still diagnostic uncertainty on the basis of clinical history and physical examination should undergo CTR without confirmatory NCS. The KSQ has a potential to decrease the duration and cost of diagnostics, reduce patient appointments and clinic time. This is especially important in busy clinics as well as during the Covid pandemic period.

\section{Conflicts of Interest}

The authors declare no conflicts of interest regarding the publication of this paper.

\section{References}

[1] BSSH (British Society for Surgery of the Hand). (2011) BSSH Evidence for Surgical Treatment (BEST): Carpal Tunnel Syndrome.

http://www.hands2elbowsurgeon.co.uk/uploads/1/5/6/1/15615196/carpal_tunnel_sy ndrome.pdf

[2] RCS (Royal College of Surgeons of England) (2017) Commissioning Guide: Treatment of Carpal Tunnel Syndrome (PDF).

https://www.rcseng.ac.uk/-/media/files/rcs/standards-and-research/commissioning/ boa--carpal-tunnel syndrome-guide-2017.pdf

[3] Gellman, H., Gelberman, R.H., Tan, A.M. and Botte, M.J. (1986) Carpal Tunnel Syndrome. An Evaluation of the Provocative Diagnostic Tests. The Journal of Bone \& Joint Surgery, 68, 735-737. https://doi.org/10.2106/00004623-198668050-00015

[4] Kamath, V. and Stothard, J. (2003) A Clinical Questionnaire for the Diagnosis of Carpal Tunnel Syndrome. Journal of Hand Surgery, 28, 455-459. https://doi.org/10.1016/S0266-7681(03)00151-7

[5] Sangram, B.S., Mayne, A.I.W. and Jariwala, A.C. (2019) Can We Accurately Predict Nerve Conduction Study Outcome Using a Carpal Tunnel Syndrome Questionnaire? Surgeon, 17, 156-159. https://doi.org/10.1016/j.surge.2019.02.001

[6] AAOS (American Academy of Orthopaedic Surgeons) (2016) Management of Carpal Tunnel Syndrome: Evidence-Based Clinical Practice Guideline. The Journal of Bone and Joint Surgery, 98, 1750-1754.

[7] Graham, B. (2008) The value added by electrodiagnostic Testing in the Diagnosis of Carpal Tunnel Syndrome. The Journal of Bone \& Joint Surgery, 90, 2587-2593. https://doi.org/10.2106/JBJS.G.01362

[8] Fowler, J.R. (2017) Nerve Conduction Studies for Carpal Tunnel Syndrome: Gold Standard or Unnecessary Evil? Orthopedics, 40, 141-142. https://doi.org/10.3928/01477447-20170419-01

[9] Franzblau, A. and Werner, R.A. (1999) What is Carpal Tunnel Syndrome? JAMA, 282, 186-187. https://doi.org/10.1001/jama.282.2.186 
[10] Homan, M.M., Franzblau, A., Werner, R.A., Albers, J.W., Armstrong, T.J. and Bromberg, M.B. (1999) Agreement between Symptom Surveys, Physical Examination Procedures and Electrodiagnostic Findings for the Carpal Tunnel Syndrome. Scandinavian Journal of Work, Environment \& Health, 25, 115-124. https://doi.org/10.5271/sjweh.413

[11] Sonoo. M., Menkes, L.D., Bland, D.P.J. and Burke, D. (2018) Nerve Conduction Studies and EMG in Carpal Tunnel Syndrome: Do they Add Value? Clinical Neurophysiology Practice, 3, 78-88. https://doi.org/10.1016/j.cnp.2018.02.005

[12] Edwards, C. and Frampton, I. (2014) Predicting the Outcome of Nerve Conduction Studies in Patients with Suspected Carpal Tunnel Syndrome: Using an Existing Carpal Tunnel Assessment Tool. Open Journal of Therapy and Rehabilitation, 2, 57-62. https://doi.org/10.4236/ojtr.2014.22010

[13] Levine, D.W., Simmons, B.P., Koris, M.J., Daltroy, L.H., Hohl, G.G., Fossel, A.H., et al. (1993) A Self-Administrated Questionnaire for the Assessment of Severity of Symptoms and Functional Status in Carpal Tunnel Syndrome. The Journal of Bone \& Joint Surgery, 75, 585-592. https://doi.org/10.2106/00004623-199311000-00002

[14] Bridges, M.J., Robertson, D.C. and Chuck, A.J. (2011) Predicting the Result of Nerve Conduction Tests in Carpal Tunnel Syndrome Using a Questionnaire. Hand Surgery, 16, 39-42. https://doi.org/10.1142/S0218810411005047

[15] Stevens, J.C. (1997) The Electrodiagnosis of Carpal Tunnel Syndrome. Muscle \& Nerve, 20, 1477-1486.

https://doi.org/10.1002/(SICI)1097-4598(199712)20:12<1477::AID-MUS1>3.0.CO;2-5

[16] Ward, M., Gruppen, L. and Regehr, G. (2002) Measuring Self-Assessment: Current State of the Art. Advances in Health Sciences Education: Theory and Practice, 7, 63-80. https://doi.org/10.1023/A:1014585522084 